\title{
Remarks Concerning the Connection between Properties of the 4-Point-Function and the Wilson-Zimmermann Expansion
}

\author{
S. Schlieder and E. Seiler \\ Max-Planck-Institut für Physik und Astrophysik, München, \\ Federal Republic of Germany
}

Received January 29, 1973

\begin{abstract}
This paper concerns an investigation of the Wilson-Zimmermann (or "short distance") expansion for $A(x) A(y)$ with $x \rightarrow y$ where $A(x)$ is a real scalar field fulfilling Wightman's axioms. If one assumes that such an expansion exists, where the terms of the expansion are operators relatively local to $A(x)$, then the singularities arising in the 4-pointfunction for $x_{3} \rightarrow x_{4}$ must control the singularities of the $n$-point functions $(n=4,5,6, \ldots)$ arising for $x_{j} \rightarrow x_{j+1}, j=1,2, \ldots, n-1$. A similar consequence can be drawn if the terms of the expansion are assumed to exist only as bilinear-forms (Section 2). For certain classes of fields one can show that this condition necessary for the short distance expansion is indeed fulfilled (Section 3). The result of the last section is that the above mentioned condition is also sufficient for the Wilson-Zimmermann expansion, interpreted as an expansion into bilinear forms, and also as an operator expansion in a somewhat modified sense.
\end{abstract}

\section{Introduction}

A field theory in which the principle of contact interaction shall be valid needs for the definition of the interaction terms products of field quantities taken at the same position.

If one intends to apply this principle also in a relativistic quantum field theory one has to define products of field operators at the same position. The difficulties which arise in this procedure are very well known. They have their origin in the distributive character of the field operators.

In the case of the free field $A_{0}(x)$ with $x=\left(x_{0}, x_{1}, x_{2}, x_{3}\right)$ however it is well known how one can get $A_{0}^{n}(x), n=2,3,4 \ldots$ in the form of Wick products of the field, by subtracting products of 2-point-functions $W_{2}^{0}$. The $A_{0}^{n}(x)$ are again tempered distributions which are relatively local to $A_{0}(x)$, this means

$$
\left[A_{0}^{n}(x), A_{0}(y)\right]=0 \quad \text { for } x-y \text { spacelike. }
$$

In the last years a heuristic ansatz for the products of field-operators at the same position in the case for interacting fields has been proposed 
especially by Wilson and Zimmermann $[1,2,3,4]$ and by Brandt [5]. For instance for a real scalar field Wilson and Zimmermann assume the expansion

$$
\begin{aligned}
A\left(x+\chi_{1}\right) A\left(x+\chi_{2}\right) \ldots A\left(x+\chi_{n}\right) & \\
& =\sum_{j=1}^{m} s_{j}\left(\chi_{1}, \ldots, \chi_{n}\right) B_{j}(x)+R_{m}\left(x ; \chi_{1}, \ldots, \chi_{n}\right) .
\end{aligned}
$$

The $B_{j}(x)$ are field operators relatively local to $A(x)$, the $s_{j}\left(\chi_{1}, \ldots, \chi_{n}\right)$ functions which become in general singular for $\chi_{j} \rightarrow 0, j=1,2, \ldots, n$, so that

$$
\lim _{\chi_{1}, \ldots, \chi_{n} \rightarrow 0} \frac{s_{j+1}\left(\chi_{1}, \ldots, \chi_{n}\right)}{s_{j}\left(\chi_{1}, \ldots, \chi_{n}\right)}=0 .
$$

With respect to the operator $R_{m}\left(x ; \chi_{1}, \ldots, \chi_{n}\right)$ it is assumed that

$$
\lim _{\chi_{1}, \ldots, \chi_{n} \rightarrow 0} \frac{R_{m}\left(x ; \chi_{1}, \ldots, \chi_{n}\right)}{s_{m}\left(\chi_{1}, \ldots, \chi_{n}\right)}=0 .
$$

Thereby the operators $B_{j}(x)$ are candidates for $A^{n}(x)$. Zimmermann [2] and Brandt [5] have proved the validity of such an expansion for certain examples in perturbation theory, Wilson and Zimmermann [3] gave conditions under which the expansion is valid and they discussed several consequences of it; furthermore Lowenstein [6] has proved the expansion rigorously for the Thirring model.

The aim of this paper is to present the first steps which must be necessarily taken to give a proof of the Wilson-Zimmermann expansion if one starts from a relativistic quantum field theory fulfilling Wightman's conditions $[7,8]$.

We consider here only the simplest case: The product of two operators for a real scalar field:

$$
A\left(x_{1}\right) A\left(x_{2}\right)=\sum_{j=1}^{m} s_{j}\left(\chi_{1}, \chi_{2}\right) B_{j}(x)+R_{m}\left(x ; \chi_{1}, \chi_{2}\right) .
$$

We intend to characterize the theory by Wightman's distributions (vacuum expectation values)

$$
\begin{aligned}
& \left(\Omega, A\left(x_{1}\right) \ldots A\left(x_{j}\right) A\left(x_{j+1}\right) \ldots A\left(x_{n}\right) \Omega\right) \\
& \quad=\mathscr{W}_{n}\left(x_{1}, \ldots, x_{j}, x_{j+1}, \ldots, x_{n}\right)=W_{n}\left(\xi_{1}, \ldots, \xi_{j}, \ldots, \xi_{n-1}\right)
\end{aligned}
$$

with $\Omega$ as invariant state under the Poincare group and with $\xi_{j}=x_{j+1}-x_{j}$.

If the expansion (1.1) with the above mentioned limiting conditions for $x_{1} \rightarrow x, x_{2} \rightarrow x$ shall be possible $\left(B_{j}(x)\right.$ are operators), one has to assume that the vacuum-expectation values

$$
W_{n}\left(\xi_{1}, \ldots, \xi_{j}, \ldots, \xi_{n-1}\right) \text { for } \xi_{j} \rightarrow 0
$$


show singularities which are essentially

a) independent of $n$

b) for fixed $n$, independent of $j$

c) for fixed $n$ and $j$ independent of the other variables.

The same statement remains correct if one interprets the result of the limiting procedure $x_{1} \rightarrow x, x_{2} \rightarrow x$ performed in (1.1) not as an equation for operators but as an equation for bilinear forms.

The essential independence of the singularities of $n$ leads especially to a connection between the singularities of $W_{4}$ and $W_{n}$ with $n>4: A$ justification of the Wilson-Zimmermann expansion is only possible, if the singularities of $W_{4}$ arising for

$$
\xi_{3} \rightarrow 0 \text { in } W_{4}\left(\xi_{1}, \xi_{2}, \xi_{3}\right)
$$

control the singularities arising for

$$
\xi_{j} \rightarrow 0 \text { in } W_{n}\left(\xi_{1}, \ldots, \xi_{j}, \ldots, \xi_{n-1}\right), \quad n>4 .
$$

In Section 2 we will prove this statement: The assumed connection between the singularities of $W_{4}$ and $W_{n}(n>4)$ is a necessary condition for the existence of the Wilson-Zimmermann expansion.

A much more difficult problem is whether one can prove by using the usual properties of $W_{n}$, that really the singularities of $W_{4}$ arising in (1.2) control those arising in (1.3) for $W_{n}, n>4$. At the moment we are only able to give a partial answer by proving this for special classes of field theories (Section 3). A generalisation of our result to more interesting cases would be important for the clarification of the situation.

In Section 4 finally we come back to the original question by showing the connection between our results concerning the singularities of $W_{n}$ and the Wilson-Zimmermann expansion. We give there a discussion of the difficulties arising if one tries to generalize the results of Section 3.

\section{Necessary Conditions for the Existence of the Wilson-Zimmermann Expansion}

According to the remarks in the introduction we intend to show in this section that the assumption of the existence of the Wilson-Zimmermann expansion implies that singularities appearing for $\xi_{j} \rightarrow 0$ in

$$
W_{n}\left(\xi_{1}, \ldots, \xi_{n-1}\right)=\left(\Omega, A\left(x_{1}\right) \ldots A\left(x_{j}\right) A\left(x_{j+1}\right) \ldots A\left(x_{n}\right) \Omega\right)
$$

have their counterparts in singularities appearing in

$$
W_{4}\left(\xi_{1}, \xi_{2}, \xi_{3}\right) \text { for } \xi_{3} \rightarrow 0 \text {. }
$$


For this one has to assume that the expansion is an expansion for operators. If it is an expansion which yields for $\xi_{j} \rightarrow 0$ only a sum of bilinear forms, then the singularities appearing for $\xi_{j} \rightarrow 0$ in $W_{n}(n>4)$ must be found again for $\xi_{1} \rightarrow 0, \xi_{3} \rightarrow 0$ simultaneously in $W_{4}\left(\xi_{1}, \xi_{2}, \xi_{3}\right)$.

Before starting the rather simple proofs for these statements we wish to repeat in short the assumptions and fix the notations.

The field $A(x)$ and the Hilbert space $\mathfrak{H}$ are assumed to fulfil Wightman's conditions; an asymptotic condition for $x_{0} \rightarrow \pm \infty$, however, is not needed at the moment.

Notations: Subspace $\mathfrak{D}$ is

$$
\begin{gathered}
\left\{\Phi \in \mathfrak{H}: \Phi=\sum_{k=0}^{n} \Phi_{k} ; \quad(n<\infty)\right. \\
\Phi_{0}=\lambda \Omega ; \Phi_{k}=\int A\left(x_{1}\right) \ldots A\left(x_{k}\right) f\left(x_{1}, \ldots, x_{k}\right) d x \\
\left.\quad f \in \mathscr{S}\left(\mathbb{R}^{4 k}\right)\right\}
\end{gathered}
$$

and $\mathfrak{D}$ is dense in $\mathfrak{H}$.

While for the construction of $\mathfrak{D}$ polynomials of the field operators of arbitrary degree have to be applied to the vacuum we need also some subspaces of $\mathfrak{D}$.

$$
\begin{gathered}
\mathfrak{D}_{l}:=\left\{\Phi \in \mathfrak{H}: \Phi=\sum_{k=0}^{n} \Phi_{k} ; \quad(n \leqq l) ;\right. \\
\left.\Phi_{0}=\lambda \Omega ; \Phi_{k}=\int A\left(x_{1}\right) \ldots A\left(x_{k}\right) f\left(x_{1}, \ldots, x_{k}\right) d x\right\} .
\end{gathered}
$$

The closure of $\mathfrak{D}_{l}$ is denoted by $\mathfrak{H}_{l}$, the corresponding projection operator by $P_{l}$.

Let us now formulate the first part of the statement more precisely.

(A) It is assumed, that the Wilson-Zimmermann expansion is valid in the form

$$
A(x+\chi) A(x-\chi)=\sum_{j=1}^{m} s_{k}(\chi) B_{j}(x)+R_{m}(x, \chi)
$$

and has the property, that

$$
\lim _{\chi \rightarrow 0} \frac{1}{s_{l}(\chi)}\left[A(x+\chi) A(x-\chi)-\sum_{j=1}^{l-1} s_{j}(\chi) B_{j}(x)\right]=B_{l}(x) .
$$

The limit is taken for a fixed direction of $\chi$, this means $\chi=\lambda \mu \lambda \geqq 0$, $\mu$ a fixed 4 -vector $\neq 0$. If the left hand side is denoted by $C_{l}(x, \chi)$, then it is assumed that for each fixed $f \in \mathscr{S}$ and each sequence $g_{j} \rightarrow \delta, g_{j} \in \mathscr{S}$,

$$
\lim _{j \rightarrow \infty}\left(\Psi, \int\left(C_{l}(x, \chi)-B_{l}(x)\right) f(x) g_{j}(\chi) d x d \chi \Phi\right)=0 .
$$

$\Psi$ arbitrary in $\mathfrak{H}, \Phi$ arbitrary in $\mathfrak{D}$. The limit of $g_{j}$ towards the Dirac measure $\delta$ is understood in the sense that for each continuous $c(x)$

$$
\lim _{j \rightarrow \infty} \int c(x) g_{j}(x) d x=c(0) .
$$


The relative locality condition is assumed in the following weak sense:

$$
\begin{gathered}
\left(\Phi, B_{l}(x) A(y) \Psi\right)=\left(A(y) \Phi, B_{l}(x) \Psi\right) \\
\Phi, \Psi \in \mathfrak{D} .
\end{gathered}
$$

Under the assumptions collected in (A) one can prove:

Lemma 1. If the Wilson-Zimmermann expansion exists and fulfils the conditions (A), then to each term $s_{k}(\chi) B_{k}(x)$ corresponds a singularity of the 4-point function $W_{4}\left(\xi_{1}, \xi_{2}, \xi_{3}\right)$ characterized by $s_{k}\left(\xi_{3}\right)$ for $\xi_{3} \rightarrow 0$.

Proof. Let us assume the contrary, for instance for the first term of the Wilson-Zimmermann expansion

$$
\lim _{\chi \rightarrow 0}\left(\Phi_{2}, C_{1}(\chi, \chi) \Omega\right)=\left(\Phi_{2}, B_{1} \Omega\right)=0
$$

for each $\Phi_{2} \in \mathfrak{D}_{2}$.

Since $B_{1} \Omega$ by (A) is a vector in $\mathfrak{H}$ it follows

$$
\left(\Phi_{2}^{\prime}, B_{1} \Omega\right)=0 \quad \text { also for } \quad \Phi_{2}^{\prime} \in \mathfrak{H}_{2} .
$$

If on the other hand $\Phi \in \mathfrak{S}$ one has

$$
\begin{gathered}
\lim _{\chi \rightarrow 0}\left(\Phi, C_{1}(x, \chi) \Omega\right)=\lim _{\chi \rightarrow 0}\left(\Phi, P_{2} C_{1}(x, \chi) \Omega\right) \\
\left(\Phi, B_{1} \Omega\right)=\left(P_{2} \Phi, B_{1} \Omega\right)=0 .
\end{gathered}
$$

From this one gets

$$
B_{1} \Omega=0 .
$$

Since by our assumptions $\Omega$ must be a separating state for $B_{1}$ it follows

$$
B_{1}=0 \text {. }
$$

This is a contradiction to its appearance in the Wilson-Zimmermann expansion. The assumptions collected in (A) guarantee that $B_{1} \Omega \in \mathfrak{H}$; so one can perform the same conclusion for the term $s_{2}(\chi) B_{2}(x)$ assuming that

$$
\lim _{\chi \rightarrow 0}\left(\Phi_{2}, C_{2}(\chi, \chi) \Omega\right)=\left(\Phi_{2}, B_{2} \Omega\right)=0 \text { for each } \Phi_{2} \in \mathfrak{D}_{2} .
$$

Repeating this procedure for each operator $B_{k}$ of the Wilson-Zimmermann expansion one sees that each singularity expressed by $s_{k}(\chi)$ must appear in the 4-point-function $W_{4}\left(\xi_{1}, \xi_{2}, \xi_{3}\right)$ for $\xi_{3} \rightarrow 0$. q.e.d.

Since in $W_{n}\left(\xi_{1}, \ldots, \xi_{j}, \ldots, \xi_{n-1}\right)$ for $\xi_{j} \rightarrow 0$ no other singularities can appear than those expressed by $s_{k}\left(\xi_{j}\right)$ - if the Wilson-Zimmermann expansion is valid in the sense of $(\mathrm{A})$ - one recognizes that it is a necessary condition for the existence of the Wilson-Zimmermann expansion, that these singularities of $W_{n}(n>4)$ are controlled by those of $W_{4}$. 
We now proceed with the second version: The Wilson-Zimmermann expansion as an expansion in bilinear forms.

(B) As in (A) the expansion shall be valid in the form (2.1) and (2.2) but instead of (2.3) here

$$
\lim _{j \rightarrow \infty}\left(\Psi, \int\left(C_{l}(x, \chi)-B_{l}(x)\right) f(x) g_{j}(\chi) d x d \chi \Phi\right)=0
$$

for $\Psi \in \mathfrak{D}$ and $\Phi \in \mathfrak{D}$.

Furthermore we make the plausible assumption that

$$
\left(A\left(x_{1}\right) \ldots A\left(x_{n}\right) \Omega, B_{l}(g) A\left(y_{1}\right) \ldots A\left(y_{m}\right) \Omega\right)
$$

are tempered distributions over $\mathbb{R}^{m+n}$.

Lemma 2. If the Wilson-Zimmermann expansion exists and fulfils the conditions (B), then the 4-point function $W_{4}\left(\xi_{1}, \xi_{2}, \xi_{3}\right)$ has a part which for $\xi_{1}, \xi_{3} \rightarrow 0$, simultaneously, becomes as singular as $\left|s_{k}(\chi)\right|^{2}$. (This means $\left\|C_{1}(x, \chi) \Omega\right\|^{2} \rightarrow 0$ for $\chi \rightarrow 0$.)

Proof. From the assumption

$$
\lim _{x \rightarrow 0}\left\|C_{1}(x, \not) \Omega\right\|^{2}=0
$$

follows not only

$$
\left(\Phi_{2}, B_{1} \Omega\right)=0 \quad \text { for } \quad \Phi_{2} \in \mathfrak{D}_{2}
$$

but also

$$
\left(\Phi_{2}^{\prime}, B_{1} \Omega\right)=0 \quad \text { for } \quad \Phi_{2}^{\prime} \in \mathfrak{H}_{2}
$$

therefore

$$
\lim _{\chi \rightarrow 0}\left(\Psi, C_{1}(x, \chi) \Omega\right)=\lim _{\chi \rightarrow 0}\left(P_{2} \Psi, C_{1}(x, \chi) \Omega\right)=0 \quad \text { for } \quad \Psi \in \mathfrak{D}
$$

or

$$
\left(\Psi, B_{1} \Omega\right)=\left(P_{2} \Psi, B_{1} \Omega\right)=0 \text { for } \Psi \in \mathfrak{D} .
$$

Using the fact that also for a bilinear form $\Omega$ is separating ${ }^{1}$ one concludes

$$
\left(\Psi, B_{1} \Phi\right)=0 \quad \text { for } \quad \Psi \in \mathfrak{D}, \Phi \in \mathfrak{D} .
$$

This is a contradiction to the appearance of $B_{1}$ in the Wilson-Zimmermann expansion. Proceeding as in the proof of Lemma 1 one can generalize this result for each $B_{l}$ of the expansion. q.e.d.

Remark. If the bilinear forms $B_{l}$ have the property that

$$
\lim _{j \rightarrow \infty}\left(\Phi_{2}^{(j)}, B_{k} \Omega\right)=\left(P_{2} \Phi, B_{k} \Omega\right)
$$

with $\Phi \in \mathfrak{D}, \Phi_{2}^{(j)} \in \mathfrak{D}_{2}$ and $\Phi_{2}^{(j)} \stackrel{s}{\rightarrow} P_{2} \Phi$ then one can prove also in case (B) the statement of Lemma 1.

1 Appendix 1. 


\section{Connection between the Singularities of the 4-and $n$-point Functions in Special Cases}

In this section we intend to show that under special assumptions concerning $W_{4}$ the conditions which according to Section 2 are necessary for the existence of the Wilson-Zimmermann expansion are fulfilled. (In Section 4 we discuss to what ex tent these conditions are sufficient for the construction of the short distance expansion (synonym for WilsonZimmermann expansion).)

Let us first introduce some notations (we follow Jost's book [8]):

$$
\begin{aligned}
\xi:= & \left(\zeta_{1}, \ldots, \zeta_{n-1}\right) \in \mathbb{C}^{4 n-4} \\
\xi:= & \left(\zeta_{1}, \ldots, \zeta_{J-1}, \zeta_{j+1}, \ldots, \zeta_{n-1}\right) \in \mathbb{C}^{4 n-8} \\
\tau_{ \pm}^{(n-1)}:= & \left\{\xi \in \mathbb{C}^{4 n-4}: \operatorname{Im} \zeta_{k} \in \mathscr{V}_{ \pm} \quad(k=1, \ldots, n-1)\right\} \\
& (\text { "forward/backward tube") } \\
\tau^{(n-1)^{\prime}}:= & \left\{\xi \in \mathbb{C}^{4 n-4}: \exists \Lambda \in \mathscr{L}_{+}(\mathbb{C})\right. \text { such that } \\
& \left.\Lambda \zeta \in \tau_{+}^{(n-1)}\right\} \\
& \text { ("extended tube") }
\end{aligned}
$$

For $\pi \in S_{n}$ (group of permutations of $n$ elements) we define

$$
\begin{aligned}
\zeta_{\pi}:= & \left(\sum_{j=1}^{\pi(2)-1} \zeta_{j}-\sum_{j=1}^{\pi(1)-1} \zeta_{j}, \ldots, \sum_{j=1}^{\pi(n)-1} \zeta_{j}-\sum_{j=1}^{\pi(n-1)-1} \zeta_{j}\right) \\
\tau_{ \pm \pi}^{(n-1)}:= & \left\{\xi \in \mathbb{C}^{4 n-4}: \zeta_{\pi} \in \tau_{ \pm}^{(n-1)}\right\} \\
& \text { ("permuted forward/backward tube") } \\
\tau_{\pi}^{(n-1)^{\prime}}:= & \left\{\xi \in \mathbb{C}^{4 n-4}: \zeta_{\pi} \in \tau^{(n-1)^{\prime}}\right\} \\
& \text { ("permuted extended tube") } \\
\Phi_{n}\left(z_{1}, \ldots, z_{n}\right):= & \int e^{i \sum_{k=1}^{n} p_{k} z_{k}} \tilde{A}\left(p_{1}\right) \ldots \tilde{A}\left(p_{n}\right) d p_{1} \ldots d p_{n} \Omega
\end{aligned}
$$

is a vector-valued holomorphic function for $z_{1} \in \tau_{+}^{(1)}$;

$$
z_{k}-z_{k-1} \in \tau_{+}^{(1)}, \quad k=2, \ldots, n
$$

(cf. Jost's book). (It is not necessary to distinguish between weak and strong analyticity; this follows from the Banach-Steinhaus theorem which states that a weakly bounded set in a Hilbert space $\mathfrak{H}$ is also strongly bounded.) We formulate now the following 
Theorem. Let us assume that a function $r(\zeta)$ exists with the following properties:

a) $r$ is holomorphic in $\tau_{+}^{(1)}$

b) $r(\zeta)=\overline{r(-\bar{\zeta})}$

c) $r$ is invariant under the (homogeneous) Lorentz group

d) $W_{4}\left(\zeta_{1}, \zeta_{2}, \zeta_{3}\right) r\left(\zeta_{3}\right)$ has a (locally unique ${ }^{2}$ ) analytic continuation to the points $\left(\zeta_{1}, \zeta_{2}\right) \in \tau_{+}^{(2)}, \zeta_{3} \in \mathscr{U}_{r}(0)$ where $\mathscr{U}_{r}(0)$ is a real neighborhood of 0 independent of $\zeta_{1}, \zeta_{2}\left(W_{4}\right.$ is the four-point function of our field theory).

e) there exists an integer $N>0$ such that for each compact subset $\mathscr{K} \subset \mathscr{V}_{+} ; \xi \in \mathscr{U}_{r}, \frac{\eta}{\sqrt{\eta^{2}}} \in \mathscr{K}, r$ fulfils the following inequality

$$
|r(\xi+i \eta)| \leqq\left(\eta^{2}\right)^{-N} C_{K} \quad\left(0<\eta^{2} \leqq 1\right)^{3} .
$$

Then $W_{n}\left(\zeta^{\prime}\right) r\left(\zeta_{j}\right)$ has a (locally unique) analytic continuation to the points $\zeta_{j}=0, \zeta_{\xi} \in \tau^{(n-2)^{\prime}}$.

The proof of this theorem will be given in three steps; each step will be formulated as a lemma.

Lemma 1. Under the assumptions of the theorem for each $\Psi \in \mathfrak{H}$ the function $\left(\Psi, \Phi_{2}(z, z+\zeta) r(\zeta)\right)$ has a (locally unique) analytic continuation to the points $z \in \tau_{+}^{(1)}, \zeta \in \mathscr{U}_{r}(0)$.

Proof. We want to use the edge of the wedge theorem. Let us define for fixed $z \in \tau_{+}^{(1)}, \Psi \in \mathfrak{S}$ :

$$
\begin{gathered}
F_{1, z}:=\left(\Psi, \Phi_{2}(z, z+\zeta)\right) r(\zeta) \\
F_{2, z}:=\left(\Psi, \Phi_{2}(z+\zeta, z)\right) r(\zeta) \\
F_{1, z} \text { is holomorphic for } \zeta \in \tau_{+}^{(1)}, \\
F_{2, z} \text { is holomorphic for }-\zeta \in \tau_{+}^{(1)} ; z+\zeta \in \tau_{+}^{(1)} .
\end{gathered}
$$

If now $F_{1, z}(\xi+i 0)=F_{2, z}(\xi-i 0)$ for $\xi \in \mathscr{U}_{r}(0)$ (in the sense of distributions), then there exists a function $F_{z}$ holomorphic for $\zeta \in \tau_{+}^{(1)} ; \zeta \in \tau_{-}^{(1)} \cap\left(\tau_{+}^{(1)}-z\right)$ and $\zeta \in \mathscr{U}_{r}(0)$, which coincides with $F_{1 . z}$ and $F_{2, z}$, respectively, in their domains of definition.

2 By this we mean that each point has a neighborhood in which the continuation is unique (single-valued).

${ }^{3}$ A sufficient condition for e) is that the boundary value $r(\xi+i 0)=\lim _{\eta \rightarrow 0, \eta \in \mathscr{Y}+} r(\xi+i \eta)$ exists as a tempered distribution. (We can assume that $\mathscr{U}_{r}(0)$ is bounded.) 
Now let us take $g \in \mathfrak{D}$ with $\operatorname{supp}(g) \subset \mathscr{U}_{r}(0)$ and define for $\eta \in V_{+}$, $\operatorname{Im}(z-\zeta) \in V_{+}$

$$
\begin{aligned}
& \Phi_{\eta}:=\int \Phi_{2}(z, z+\xi+i \eta) r(\xi+i \eta) g(\xi) d \xi \\
& \Phi_{-\eta}:=\int \Phi_{2}(z+\xi-i \eta, z) r(\xi-i \eta) g(\xi) d \xi \\
& \Phi_{+}:=s-\lim _{\substack{\eta \rightarrow 0 \\
\eta \in \mathscr{V}+}} \Phi_{\eta}
\end{aligned}
$$

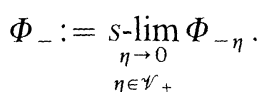

(In Appendix 2 we show that the existence of these limits follows from assumption e).)

Then

$$
\begin{aligned}
& \quad\left|\int F_{1, z}(\xi+i 0) g(\xi) d \xi-\int F_{2, z}(\xi-i 0) g(\xi) d \xi\right| \\
& =\left|\left(\Psi, \Phi_{+}-\Phi_{-}\right)\right| \leqq\|\Psi\|\left\|\Phi_{+}-\Phi_{-}\right\| \\
& \left\|\Phi_{+}-\Phi_{-}\right\|^{2}=\left(\Phi_{+}, \Phi_{+}-\Phi_{-}\right)-\left(\Phi_{-}, \Phi_{+}-\Phi_{-}\right) \\
& =\lim _{\substack{\eta \rightarrow 0 \\
\eta \in V_{+}}}\left(\Phi_{\eta}, \Phi_{+}-\Phi_{-}\right)-\lim _{\substack{\eta \rightarrow 0 \\
\eta \in V_{+}}}\left(\Phi_{-\eta}, \Phi_{+}-\Phi_{-}\right) .
\end{aligned}
$$

Each of these two terms vanishes because of assumption d):

$$
\begin{aligned}
\left(\Phi_{\eta}, \Phi_{+}-\Phi_{-}\right)=\int \overline{r\left(\xi^{\prime}+i \eta\right)}\left[\mathscr{W}_{4}\left(\bar{z}+\xi^{\prime}-i \eta, \bar{z}, z, z+\xi+i 0\right)\right. \\
\left.\quad \cdot r(\xi+i 0)-\mathscr{W}^{4}\left(\bar{z}+\xi^{\prime}-i \eta, \bar{z}, z+\xi-i 0, z\right) r(\xi-i 0)\right] \\
\quad \cdot \overline{g\left(\xi^{\prime}\right)} g(\xi) d \xi^{\prime} d \xi \\
=\int \overline{r\left(\xi^{\prime}+i \eta\right)}\left[\mathscr{W}_{4}\left(\bar{z}+\xi^{\prime}-i \eta, \bar{z}, z, z+\xi+i 0\right) r(\xi+i 0)\right. \\
\left.\quad-\mathscr{W}_{4}\left(\bar{z}+\xi^{\prime}-i \eta, \bar{z}, z, z+\xi-i 0\right) r(\xi-i 0)\right] \overline{g\left(\xi^{\prime}\right)} g(\xi) d \xi^{\prime} d \xi \\
=\int \overline{r\left(\xi^{\prime}+i \eta\right)}\left[W_{4}\left(-\xi^{\prime}+i \eta, 2 i \operatorname{Im} z, \xi+i 0\right) r(\xi+i 0)\right. \\
\left.\quad-W_{4}\left(-\xi^{\prime}+i \eta, 2 i \operatorname{Im} z, \xi-i 0\right) r(\xi-i 0)\right] \overline{g\left(\xi^{\prime}\right)} g(\xi) d \xi^{\prime} d \xi \\
=0
\end{aligned}
$$

(in the second step we used locality). The vanishing of the second term can be seen similarly. Hence

$$
F_{1, z}(\xi+i 0)=F_{2, z}(\xi-i 0) \quad\left(\xi \in \mathscr{U}_{r}(0)\right)
$$

and there is a function $F_{z}(\zeta)$ as described above. From the generalized Hartogs' theorem (see Vladimirov [9], $\S 20.6^{4}$ ) we can infer that $F_{z}(\zeta)$ is holomorphic with respect to $(z, \zeta)$ at the points $z \in \tau_{+}^{(1)}, \zeta \in \mathscr{U}_{r}(0)$.

For the sake of completeness we prove the following corollary:

Corollary. $\Phi_{2}(z, z+\zeta) r(\zeta)$ can be continued as a vector-valued analytic function of $(z, \zeta)$ to the points $z \in \tau_{+}^{(1)}, \zeta \in \mathscr{U}_{r}(0)$.

${ }_{4}$ The English translation contains a misprint in this theorem. 
Proof. The corollary follows from Lemma 1 and the following general statement:

Proposition. If a vector-valued holomorphic function $\Phi: G \subset \mathbb{C}^{n} \rightarrow \mathfrak{H}$ (Hilbert space) is such that for every $\Psi \in \mathfrak{H}$ the function $(\Psi, \Phi(z))$ can be analytically continued into some larger domain $\tilde{G} \supset G, \Phi$ can be continued as a vector-valued analytic function into $\tilde{G}$.

Proof of the Proposition. From the convergence of the Taylor expansion of $(\Psi, \Phi(z))$ for every $\Psi \in \mathfrak{H}$ follows the weak convergence of the corresponding expansion of $\Phi(z)$ (because every Hilbert space is weakly complete). Therefore $\Phi(z)$ is analytic in $\tilde{G}$ in the weak sense; according to our previous remark this is equivalent to strong analyticity.

Lemma 2. Under the assumptions of the theorem $W_{n}\left(\zeta_{1}, \ldots, \zeta_{n-1}\right) r\left(\zeta_{n-1}\right)$ has a (locally unique) analytic continuation to the points $\left(\zeta_{1}, \ldots, \zeta_{n-2}\right)$ $\in \tau^{(n-2)^{\prime}}$ (extended tube), $\zeta_{n-1}=0$.

Proof. We use Lemma 1 for

$$
\Psi:=\Phi_{n-2}\left(\bar{z}_{n-2}, \ldots, \bar{z}_{1}\right)
$$

(with the usual restriction on the arguments); we learn that

$$
\begin{aligned}
\left(\Psi, \Phi_{2}\left(z_{n-1},\right.\right. & \left.\left.z_{n-1}+\zeta_{n-1}\right)\right) r\left(\zeta_{n-1}\right) \\
& =\mathscr{W}_{n}\left(z_{1}, \ldots, z_{n-1}, z_{n-1}+\zeta_{n-1}\right) r\left(\zeta_{n-1}\right) \\
& =W_{n}\left(\zeta_{1}, \ldots, \zeta_{n-1}\right) r\left(\zeta_{n-1}\right)
\end{aligned}
$$

can be analytically continued for fixed $\left(z_{1}, \ldots, z_{n-2}\right)$ into some neighborhood $\mathscr{V}$ of $\tau_{+}^{(1)} \times \mathscr{U}_{r}(0)$. On the other hand $\mathscr{W}_{n} \cdot r$ is holomorphic with respect to $z_{1}, \ldots, z_{n-2}$ for fixed $\left(z_{n-1}, \zeta_{n-1}\right) \in \mathscr{V}$ if $\zeta_{k}=z_{k+1}-z_{k} \in \tau_{+}^{(1)}$ $(k=1, \ldots, n-2)$ and $z_{n-2} \in \tau_{-}^{(1)}$; that means that also $W_{n} \cdot r$ is holomorphic with respect to $\left(\zeta_{1}, \ldots, \zeta_{n-2}\right) \in \tau_{+}^{(n-2)}\left(z_{n-1}-\zeta_{n-2} \in \tau_{-}^{(1)}\right)$ for fixed $\left(z_{n-1}, \zeta_{n-1}\right) \in \mathscr{V}$; by Hartogs' main theorem it is holomorphic with respect to $\left(\zeta_{1}, \ldots, \zeta_{n-1}\right)$, if $\left(\zeta_{1}, \ldots, \zeta_{n-2}\right) \in \tau_{+}^{(n-2)}, z_{n-1}-\zeta_{n-2} \in \tau_{-}^{(1)}$, $\left(z_{n-1}, \zeta_{n-1}\right) \in \mathscr{V}$.

Since $W_{n} \cdot r$ actually does not depend on $z_{n-1}$, we can conclude that it is holomorphic for $\left(\zeta_{1}, \ldots, \zeta_{n-2}\right) \in \tau_{+}^{(n-2)}, \zeta_{n-1} \in \mathbb{U}_{r}(0)$; Lemma 2 follows if we take into account the Lorentz invariance of $W_{n}$ and $r$.

From Lemma 2 we can already deduce a statement about the holomorphy of $r\left(\zeta_{j}\right) W_{n}\left(\zeta_{1}, \ldots, \zeta_{n-1}\right)$ at $\zeta_{j}=0$, if we take into account the locality condition. Locality tells us

$$
\begin{aligned}
& r\left(\zeta_{j}\right) W_{n}\left(\zeta_{1}, \ldots, \zeta_{n-1}\right) \\
& =r\left(\zeta_{j}\right) W_{n}\left(\zeta_{1}, \ldots, \zeta_{j-2}, \zeta_{j-1}+\zeta_{j}+\zeta_{j+1}, \zeta_{j+2}, \ldots, \zeta_{n-1},-\sum_{l=j}^{n-1} \zeta_{l}, \zeta_{j}\right) .
\end{aligned}
$$


We know from Lemma 2 that the right hand side is holomorphic at the points

$$
\zeta_{j}=0,\left(\zeta_{1}, \ldots, \zeta_{j-2}, \zeta_{j-1}+\zeta_{j+1}, \zeta_{j+2}, \ldots, \zeta_{n-1},-\sum_{l=j+1}^{n-1} \zeta_{l}\right) \in \tau^{(n-2) \prime} .
$$

If we define

$$
\begin{gathered}
\tau_{\tilde{\pi}}^{(n-2)^{\prime}}:=\left\{\left(\zeta_{1}, \ldots, \zeta_{j-1}, \zeta_{j+1}, \ldots, \zeta_{n-1}\right)=\xi_{\mathrm{z}} \in \mathbb{C}^{4 n-8}:\right. \\
\left.:\left(\zeta_{1}, \ldots, \zeta_{j-2}, \zeta_{j-1}+\zeta_{j+1}, \zeta_{j+2}, \ldots, \zeta_{n-1},-\sum_{l=j+1}^{n-1} \zeta_{l}\right) \in \tau^{(n-2) \prime}\right\}
\end{gathered}
$$

(this is a permuted extended tube)

we can restate the result of Lemma 2 in the form $r\left(\zeta_{j}\right) W_{n}\left(\zeta_{1}, \ldots, \zeta_{n-1}\right)$ is holomorphic for $\zeta_{j}=0$,

$$
\left.\xi=\left(\zeta_{1}, \ldots, \zeta_{j-1}, \zeta_{j+1}, \ldots, \zeta_{n-1}\right) \in \tau_{\hat{\pi}}^{(n-2}\right)^{\prime} .
$$

It is well known (cf. for instance the book of Streater and Wightman) [7] that

$$
\mathscr{H}:=\tau_{+}^{(n-2)} \cap \tau_{\hat{\pi}}^{(n-2) \prime}
$$

is not empty (it is clearly open). What remains to be done is to extend the holomorphy of $r\left(\zeta_{j}\right) W_{n}(\xi)$ from the points $\zeta_{j}=0$, $\xi_{\xi} \in \mathscr{H}$ to all points $\zeta_{j}=0, \xi_{\xi} \in \tau_{+}^{(n-2)}$.

One possibility to achieve this is to use the pseudoconvexity property of domains of holomorphy; this turns out to be rather complicated and one has to be very careful to guarantee the single-valuedness of the continuation. We can choose here an easier method because we have the very advanced tool of the edge of the wedge theorem at hand.

Lemma 3. Let $F\left(\zeta_{1}, \ldots, \zeta_{n-1}\right)$ be holomorphic in $\mathscr{D}=\tau_{+}^{(n-1)} \cup \tau_{+\pi}^{(n-1)}$ where $\tau_{+\pi}^{(n-1)}=\left\{\tilde{\hbar} \in \mathbb{C}^{4 n-4}:\left(\zeta_{1}, \ldots, \zeta_{j-2}, \zeta_{j-1}+\zeta_{j},-\zeta_{j}, \zeta_{j+1}+\zeta_{j}, \ldots, \zeta_{n-1}\right)\right.$ $\in \tau_{\pi}^{(n-1)}$, and in the points $\zeta_{j}=0, \xi \in \mathscr{H}\left(\tau_{+}^{(n-2)}\right.$ ( $\mathscr{H}$ open and not empty). Assume that the boundary values

$$
F\left(\zeta_{1}, \ldots, \zeta_{j-1}, \zeta_{j} \pm i 0, \zeta_{j+1}, \ldots, \zeta_{n-1}\right)
$$

exist as distributions ${ }^{5}$ in $\xi_{j}$ depending holomorphically on $\xi_{\xi} \in \tau_{+}^{(n-2)}$. Then $F$ has a (locally unique) analytic continuation to the points $\zeta_{j} \in \mathscr{V}_{e}(0)$, $\left(\zeta_{1}, \ldots, \zeta_{j-1}, \zeta_{j+1}, \ldots, \zeta_{n-1}\right) \in \tau_{+}^{(n-2)}$, where $\mathscr{V}_{\varrho}(0)$ is some real neighborhood of $0 \in \mathbb{C}^{4}$.

Proof. We choose a subdomain $\mathscr{H}_{1} \subset \mathrm{C} \mathscr{H}$ (that means $\overline{\mathscr{H}}_{1} \subset \mathscr{H}$ and $\overline{\mathscr{H}}_{1}$ compact). Then there exists a $\varrho>0$ such that $F$ is holomorphic for $\xi \in \overline{\mathscr{H}}_{1}$ and $\left|\zeta_{j}\right|<\varrho("|\ldots|$ " denotes the euclidean norm) [Proof: $F$ is holomorphic in an open neighborhood $\mathscr{N}$ of the compact set

$$
\mathscr{K}:=\left\{\xi \in \mathbb{C}^{4 n-4}: \zeta_{j}=0, \xi \in \overline{\mathscr{H}}_{1}\right\} .
$$

${ }^{5}$ Over $\mathscr{Z}\left(\mathscr{F}_{Q}(0)\right)$. 
We take for $\varrho$ the finite distance between $\mathscr{K}$ and the boundary of $\mathscr{N}]$. We define now

$$
\mathscr{V}_{\varrho}:=\left\{\zeta_{j} \in \mathbb{C}^{4}: \operatorname{Im} \zeta_{j}=0:\left|\zeta_{j}\right|<\varrho\right\} .
$$

Let us take a test function $g \in \mathscr{S}\left(\mathbb{R}^{4}\right)$ with $\operatorname{supp}(g) \subset \mathscr{V}_{\varrho}(0)$ and define

$$
G_{ \pm}\left(\xi_{)}\right):=\int F\left(\zeta_{1}, \ldots, \zeta_{j-1}, \xi_{j} \pm i 0, \zeta_{j+1}, \ldots, \zeta_{n-1}\right) g\left(\xi_{j}\right) d \xi_{j} .
$$

According to our assumptions $G_{ \pm}$are holomorphic functions of $\xi \in \tau_{+}^{(n-2)}$. For $\xi \in \mathscr{H}_{1}$ we have

Therefore

$$
G_{+}(\xi)-G_{-}\left(\xi_{\xi}\right)=0 \text {. }
$$

$$
G_{+}\left(\xi_{\xi}\right)-G_{-}\left(\xi_{5}\right)=0 \text { in } \tau_{+}^{(n-2)} .
$$

The edge of the wedge theorem tells us then that for fixed $\xi_{4} \in \tau_{+}^{(n-2)}$ $F\left(\zeta_{1}, \ldots, \zeta_{n-1}\right)$ can be analytically continued to the points $\zeta_{j} \in \mathscr{V}_{\varrho}(0)$. We use again the generalized Hartogs' theorem (Vladimirov [9], § 20.6) to infer that $F$ is holomorphic with respect to all variables at the points $\zeta_{j} \in \mathscr{V}_{\varrho}(0), \zeta_{\zeta} \in \tau_{+}^{(n-2)}$. This proves Lemma 3 .

To prove now our theorem we have to show that $F\left(\zeta_{1}, \ldots, \zeta_{n-1}\right)$ $=r\left(\zeta_{j}\right) W_{n}\left(\zeta_{1}, \ldots, \zeta_{n-1}\right)$ fulfils the assumptions of Lemma 3 . The only point we have to consider concerns the boundary values (the other assumptions have been proved above).

$F\left(\zeta_{1}, \ldots, \zeta_{j-1}, \xi_{j}+i 0, \zeta_{j+1}, \ldots, \zeta_{n-1}\right)$ is a distribution over $\mathscr{D}(\hat{\mathscr{U}})^{6}$ according to Appendix 2 (with respect to $\xi_{j}$ ). The holomorphic dependence on $\xi \in \tau_{+}^{(n-2)}$ follows from the spectrum condition. For the other boundary value we have, using locality:

$$
\begin{aligned}
& F\left(\zeta_{1}, \ldots, \zeta_{j-1}, \xi_{j}-i 0, \zeta_{j+1}, \ldots, \zeta_{n-1}\right) \\
= & F\left(\zeta_{1}, \ldots, \zeta_{j-2}, \zeta_{j-1}+\zeta_{j},-\xi_{j}+i 0, \zeta_{j+1}+\xi_{j}, \ldots, \zeta_{n-1}\right) .
\end{aligned}
$$

Hence the spectrum condition guarantees again the holomorphic dependence on $\xi \in \tau_{+}^{(n-2)}$.

Thus we see that $r\left(\zeta_{j}\right) W_{n}\left(\zeta_{1}, \ldots, \zeta_{n-1}\right)$ has a locally unique analytic continuation to the points $\zeta_{j}=0$, $\zeta_{\zeta} \in \tau_{+}^{(n-2)}$; by using Lorentz invariance, we can replace $\tau_{+}^{(n-2)}$ by $\tau^{(n-2)}$. This proves our theorem.

Remark. We should say something about the global single-valuedness of our continuations. This problem is answered by the following

Lemma 4. Any domain

$$
\begin{gathered}
\mathscr{D}_{n, j}:=\bigcup_{\pi \in M_{j} \subset S_{k}} \tau_{\pi}^{(n-1)^{\prime}} \cup\left\{\zeta \in \mathbb{C}^{4 n-4}:\left(\zeta_{1}, \ldots, \zeta_{j-1}\right) \in \bigcup_{\pi \in S_{j}} \tau_{\pi}^{(j-1)^{\prime}},\right. \\
\left.\left(\zeta_{j+1}, \ldots, \zeta_{n-1}\right) \in \bigcup_{\pi \in S_{n-j}} \tau_{\pi}^{(n-1-j)},\left|\zeta_{j}\right|<\varrho(\zeta)\right\} \text { is simply connected }
\end{gathered}
$$

$6 \hat{\mathscr{U}}$ denotes some real netghborhood of $0 ; \mathscr{D}(\hat{\mathscr{U}})$ is the space of all $C^{\times}$-functions with compact support in $\hat{\mathscr{U}}$. 
Thereby $S_{n}$ is the symmetric group of $n$ elements and $M_{j}$ is the subset of $S_{n}$ which lets the neighboring elements $j-1$ and $j$ remain neighboring; $\varrho\left(\xi_{)}\right)$ is a continuous positive function.

Consequence of Lemma 4. If a function is holomorphic and singlevalued in $\bigcup_{\pi \in M_{j}} \tau_{\pi}^{(n-1)^{\prime}}$ and if it can be analytically continued to every point of $\mathscr{D}_{n, j}$, the continuation is single-valued (theorem of monodromy). If we specialize this result to $n=j=4$ it follows that we do not need the words "locally unique" in assumption d) of the theorem.

Proof of Lemma 4. For $0 \leqq \lambda \leqq 1$ we define a set of mappings

by

$$
\begin{gathered}
\omega_{\lambda}: \mathscr{D}_{n, j} \rightarrow \mathscr{D}_{n, j} \\
\omega_{\lambda}(\zeta):=\left(\zeta_{1}, \ldots, \zeta_{j-1}, \lambda \zeta_{j}, \zeta_{j+1}, \ldots, \zeta_{n-1}\right) .
\end{gathered}
$$

By use of these mappings we can construct a homotopy of every closed curve

to the closed curves

$$
S:[0,1] \rightarrow \mathscr{D}_{n, j}
$$

especially to

$$
\begin{aligned}
& s_{\lambda_{\lambda}}:=\omega_{\lambda} \cdot s \\
& s_{0}=\omega_{0} \cdot s
\end{aligned}
$$

$s_{0}$ lies completely in the set

$$
\begin{gathered}
\left\{\xi \in \mathbb{C}^{4 n-4}: \zeta_{J}=0 ;\left(\zeta_{1}, \ldots, \zeta_{j-1}\right) \in \bigcup_{\pi \in S} \tau_{\pi}^{(j-1)^{\prime}},\right. \\
\left.\left(\zeta_{j+1}, \ldots, \zeta_{n-1}\right) \in \bigcup_{\pi \in S_{n-j}} \tau_{\pi}^{(n-j-1)^{\prime}}\right\}
\end{gathered}
$$

which is simply connected according to a theorem by Tomozawa [10]. Hence $s$ can be contracted to a point, which proves Lemma 4.

Conclusion. Under the assumptions of the theorem the function $F(\xi)=r\left(\zeta_{n-1}\right) W_{n}(\xi)$ has a unique analytic continuation to the points $\zeta_{n-1}=0,\left(\zeta_{1}, \ldots, \zeta_{n-2}\right) \in \bigcup_{\pi \in S_{n-1}} \tau_{\pi}^{(n-2), 7}$. This has the consequence that we have a Taylor expansion (with a finite range of convergence):

$$
F(\zeta)=\sum_{N} \zeta_{n-1}^{N} F_{N}\left(\zeta_{1}, \ldots, \zeta_{n-2}\right)
$$

where the "coefficients" $F_{N}$ are holomorphic in $\bigcup_{\pi \in S_{n-1}} \tau_{\pi}^{(n-2) \prime}$. In the next section we discuss how far the functions $F_{N}$ can be interpreted as vacuum expectation values containing the composite operators of the short distance expansion.

7 This fact implies that $W_{n}$ shows the same singularity whenever two consecutive arguments coincide: this is one of the assumptions made by Genz and de Motton [11] for their proof that the singularity of the two-point function is the dominating one. 


\section{Application of the Results to the Short Distance Expansion; Question of Generalisations}

Two essentially different subjects are contained in this section.

The first one concerns the connection between the statement of the theorem of Section 3 and the short distance expansion. The second one refers to the question how one should try to formulate generalisations of this theorem. We intend to show the difficulties which one has to overcome if one tries to prove these generalisations.

Let us begin with the first part. We know that with our assumptions

$$
W_{n}\left(\zeta_{1}, \ldots, \zeta_{n-1}\right) r\left(\zeta_{j}\right), \quad n=2,3,4, \ldots
$$

is analytic in the points $\zeta_{j}=0$, 安 $\in \tau^{(n-2)^{\prime}}$. As a consequence of this result we can formulate

Lemma 1. The Wilson-Zimmermann expansion

$$
A(x+\chi) A(x-\chi)=\sum_{j=1}^{m} s_{k}(\chi) B_{k}(x)+R_{m}(x, \chi)
$$

exists as an expansion in bilinear forms (for the definition of $C_{l}(x, \chi)$ compare (2.3)):

$$
\lim _{j \rightarrow \infty}\left(\Psi_{1}, \int\left[C_{l}(x, \chi)-B_{l}(x)\right] f(x) g_{j}(\chi) d x d \chi \Psi_{2}\right)=0
$$

$\Psi_{1} \in \mathfrak{J}, \Psi_{2} \in \mathfrak{J}$; $\mathfrak{J}$ is the linear hull of the vectors (compare (3.8))

with

$$
\{\Omega\},\left\{\Phi_{1}\left(z_{1}\right)\right\},\left\{\Phi_{2}\left(z_{1}^{\prime}, z_{2}^{\prime}\right)\right\},\left\{\Phi_{3}\left(z_{1}^{\prime \prime}, z_{2}^{\prime \prime}, z_{3}^{\prime \prime}\right)\right\}, \ldots
$$

$z_{1} \in \tau_{+}^{(1)} ; z_{1}^{\prime} \in \tau_{+}^{(1)} ; z_{2}^{\prime}-z_{1}^{\prime} \in \tau_{+}^{(1)} ; z_{1}^{\prime \prime} \in \tau_{+}^{(1)}, z_{2}^{\prime \prime}-z_{1}^{\prime \prime} \in \tau_{+}^{(1)}, z_{3}^{\prime \prime}-z_{2}^{\prime \prime} \in \tau_{+}^{(1)} ; \ldots$

$\mathfrak{J}$ is dense in $\mathfrak{D}$ ( for it is constructed with testfunctions of $\mathscr{S}$ exponentially decreasing in momentum space) and therefore dense in $\mathfrak{H}$.

Proof of Lemma 1. One can write

with

$$
\Phi_{k}\left(z_{1}, \ldots, z_{k}\right)=A\left(z_{1}\right) \Phi_{k-1}\left(z_{2}, \ldots, z_{k}\right)
$$

$$
A\left(z_{1}\right)=\int e^{l p z_{1}} \tilde{A}\left(p_{1}\right) d p_{1} .
$$

With $z_{1} \in \tau_{+}^{(1)} ; z_{j}-z_{J-1} \in \tau_{+}^{(1)}, j=2, \ldots, k, \Phi_{k-1}$ is in the domain of $A\left(z_{1}\right)$; from the identity $A\left(z_{1}\right)^{*}=A\left(\bar{z}_{1}\right)$ (this equation does not take into account the domains, which follow from the remark above) one finds

with

$$
\begin{gathered}
\left(\Phi_{j}\left(z_{1}^{\prime}, \ldots, z_{j}^{\prime}\right), \Phi_{n-j}\left(z_{1}, \ldots, z_{n-j}\right)\right) \\
=\left(\Phi_{j-1}\left(z_{2}^{\prime}, \ldots, z_{j}^{\prime}\right), A\left(\bar{z}_{1}^{\prime}\right) A\left(z_{1}\right) \Phi_{n-j-1}\left(z_{2}, \ldots, z_{n-j}\right)\right) \\
z_{1}^{\prime} \in \tau_{+}^{(1)} ; z_{l}^{\prime}-z_{l-1}^{\prime} \in \tau_{+}^{(1)}, \quad i=2, \ldots, j \\
z_{1} \in \tau_{+}^{(1)} ; z_{l}-z_{i-1} \in \tau_{+}^{(1)}, \quad i=2, \ldots, n-j .
\end{gathered}
$$


On the other hand we can write

with

$$
\left(\Phi_{j}\left(z_{1}^{\prime}, \ldots, z_{j}^{\prime}\right), \Phi_{n-j}\left(z_{1}, \ldots, z_{n-j}\right)\right)=W_{n}\left(\zeta_{1}, \ldots, \zeta_{j}, \ldots, \zeta_{n-1}\right)
$$

$$
\begin{array}{ll}
\zeta_{k}=\bar{z}_{j-k}^{\prime}-\bar{z}_{j+1-k}^{\prime}, & k=1, \ldots, j-1 \\
\zeta_{j}=z_{1}-\bar{z}_{1}^{\prime} & \\
\zeta_{k}=z_{k+1-1}-z_{k-\jmath}, & k=j+1, \ldots, n-1 .
\end{array}
$$

The theorem of Section 3 ensures us that

$$
W_{n}\left(\zeta_{1}, \ldots, \zeta_{j}, \ldots, \zeta_{n-1}\right) r\left(\zeta_{j}\right)
$$

is analytic at $\zeta_{j}=0, \zeta \in \tau_{+}^{(n-2)}$.

So we can go over from

with

$$
\left(\Phi_{j-1}\left(z_{2}^{\prime}, \ldots, z_{j}^{\prime}\right), A\left(\bar{z}_{1}^{\prime}\right) A\left(z_{1}\right) \Phi_{n-j-1}\left(z_{2}, \ldots, z_{n-j}\right)\right) r\left(\bar{z}_{1}^{\prime}-z_{1}\right)
$$

$$
\begin{array}{ll}
z_{1}^{\prime} \in \tau_{+}^{(1)} ; z_{i}^{\prime}-z_{i-1}^{\prime} \in \tau_{+}^{(1)}, & i=2, \ldots, j \\
z_{1} \in \tau_{+}^{(1)} ; z_{i}-z_{l-1} \in \tau_{+}^{(1)}, & i=2, \ldots, n-j
\end{array}
$$

to

$$
\begin{gathered}
\left(\Phi_{J-1}\left(z_{2}^{\prime}, \ldots, z_{j}^{\prime}\right), A\left(x_{1}^{\prime}\right) A\left(x_{1}\right) \Phi_{n-j-1}\left(z_{2}, \ldots, z_{n-j}\right)\right) r\left(x_{1}-x_{1}^{\prime}\right) \\
=F_{n-1}\left(\zeta_{1}, \ldots, \zeta_{j-1}, \xi_{j}, \zeta_{j+1}, \ldots, \zeta_{n-1}\right)
\end{gathered}
$$

where $F$ is defined by the left hand side of the equation, and $\xi_{j}=x_{1}-x_{1}^{\prime}$ must be in a certain neighborhood of 0 (compare Lemma 3). If $z_{1}^{\prime}$ and $z_{1}$ are chosen in this manner we can enlarge the domain of the other variables from that of (4.2) to

$$
\begin{array}{ll}
z_{2}^{\prime} \in \tau_{+}^{(1)} ; z_{l}^{\prime}-z_{i-1}^{\prime} \in \tau_{+}^{(1)}, & i=3, \ldots, j \\
z_{2} \in \tau_{+}^{(1)} ; z_{i}-z_{1-1} \in \tau_{+}^{(1)}, & i=3, \ldots, n-j .
\end{array}
$$

Therefore $\Phi_{j-1}$ and $\Phi_{n-j-1}$ in (4.3) can vary over the whole sets of states defined by (3.8) for $j-1$ or $n-j-1$ arguments, respectively. Since $F_{n-1}$ is analytic at $\xi_{j}=0$ we can expand it into a Taylor series. We intend to write this Taylor expansion in a form which can be compared with the Wilson-Zimmermann expansion; to this aim we define

$$
F_{n}\left(\zeta_{1}, \ldots, \zeta_{j-1}, \zeta_{j}, \zeta_{j+1}, \ldots, \zeta_{n-1}\right)=: \hat{F}\left(\Phi_{j-1}, \Phi_{n-j-1} ; x, \xi\right)
$$

with $\xi_{J}=x_{1}-x_{1}^{\prime}($ compare $(4.3))$ and

$$
x_{1}=x+\frac{\xi}{2}, \quad x_{1}^{\prime}=x-\frac{\xi}{2} .
$$

From the spacial states $\Phi_{j-1}, \Phi_{n-j-1}$ we can go over to an arbitrary pair of states $\Psi_{1}, \Psi_{2} \in \mathfrak{J}$ : 
$\hat{F}\left(\Psi_{1}, \Psi_{2} ; x, \xi\right)$ is therefore a bilinear form defined on $\overline{\mathfrak{J}} \times \mathfrak{I}$, depending on $x$ and $\xi$. For a sufficiently small value of $|\xi|$ the Taylor expansion

$$
\begin{aligned}
& \hat{F}\left(\Psi_{1}, \Psi_{2} ; x, \xi\right)=\sum_{\substack{n=0 \\
\mu_{k}=0, \ldots, 3}}^{\infty} \frac{1}{n !} \xi^{\mu_{1}} \ldots \xi^{\mu_{n}} \frac{\partial}{\partial \xi^{\mu_{1}}} \ldots \frac{\partial}{\partial \xi^{\mu_{n}}} \hat{F}\left(\Psi_{1}, \Psi_{2} ; x, 0\right) \\
& \text { converges. }
\end{aligned}
$$

The comparison with the short distance expansion as formulated in (2.2) leads for instance for the first term to ${ }^{8}$

$$
\lim _{\chi \rightarrow 0}\left(\Psi_{1}, \frac{A(x+\chi) A(x-\chi)}{S_{1}(\chi)} \Psi_{2}\right)=\left(\Psi_{1}, B_{1}(x) \Psi_{2}\right)=\hat{F}\left(\Psi_{1}, \Psi_{2} ; x, 0\right) .
$$

As is well known (compare Ref. [4]) the operators $B_{j}$ depend in general on the direction of $\chi$ or $\xi$, respectively, if one performs the limit $\xi \rightarrow 0$ by a sequence $\xi=\lambda_{j} \hat{\xi}$ with $\hat{\xi}$ fixed and $\lambda_{j} \rightarrow 0$ ( $\lambda_{j}$ real numbers). This fact finds its expression in the form of the general term of the expansion:

$$
\left(\Psi_{1}, B_{k}(x) \Psi_{2}\right)=\frac{1}{(k-1) !} \sum \hat{\zeta}^{\mu_{1}} \ldots \hat{\xi}^{\mu_{k-1}} \frac{\partial}{\partial \xi^{\mu_{1}}} \ldots \frac{\partial}{\partial \xi^{\mu_{k-1}}} \hat{F}\left(\Psi_{1}, \Psi_{2} ; x, 0\right)
$$

with a directional dependence of the same kind as in Ref. [4]. $R_{m}$ is represented by the remainder term of the Taylor expansion.

The comparison of the terms of the Wilson-Zimmermann expansion with those of the Taylor expansion of $\hat{F}$ completes the proof of lemma.

Remark. The bilinear forms defined by the derivatives of $\hat{F}$ are $C^{\infty}$-functions of $x$ (smearing is not necessary). This is also true for $A(x)$ considered as bilinear form on $\overline{\mathfrak{J}} \times \mathfrak{J}$ (or $\overline{\mathfrak{D}} \times \mathfrak{D}$ ) for instance.

The theorem of Section 3 enables us also to get a certain operator version of the short distance expansion. To this aim we formulate

Lemma 2. The Wilson-Zimmermann expansion is valid in the form

$$
A(z+\chi) A(z-\chi)=\sum_{k=1}^{m} s_{k}(\chi) B_{k}(z)+R_{m}(z, \chi)
$$

with $z \in \tau_{+}^{(1)}$; the $B_{k}(z)$ are defined on a dense subspace of $\mathfrak{I}$ denoted by $\mathfrak{I}_{y} ; \mathfrak{I}_{y}$ depends on $y=\operatorname{Im} z$. $\left(\mathfrak{I}_{y}=e^{-P y} \mathfrak{I}\right)$.

Remark. As before $A(z)$ is a short notation for $\int e^{l p z} \tilde{A}(p) d p ;$ since $\mathfrak{I}$ is dense in $\mathfrak{H}, \mathfrak{J}_{y}$ is dense in $\mathfrak{H} ; \mathfrak{J}_{y}$ is invariant under translations.

Proof of Lemma 2. For fixed $z^{\prime}, z_{1}, \ldots, z_{k}$ with

$$
\operatorname{Im} z^{\prime} \in \mathscr{V}_{+}, \operatorname{Im}\left(z_{1}-z^{\prime}\right) \in \mathscr{V}_{+}, \operatorname{Im}\left(z_{j}-z_{j-1}\right) \in \mathscr{V}_{+} \quad(j=2, \ldots, k)
$$

${ }^{8}$ If $\hat{F}\left(\Psi_{1}, \Psi_{2}: x, 0\right)=0$ we take instead of it the first nonvanishing derivative of $F$ with respect to $\xi$ (at $\xi=0$ ). 
we consider the vector-valued holomorphic functions

$$
\begin{aligned}
\Psi_{1}(\zeta):= & r(\zeta) A\left(z^{\prime}\right) A\left(z^{\prime}+\zeta\right) A\left(z_{1}\right) \ldots A\left(z_{k}\right) \Omega \\
& \left(\operatorname{Im} \zeta \in \mathscr{V}_{+}, \operatorname{Im}\left(z_{1}-z^{\prime}-\zeta\right) \in \mathscr{V}_{+}\right) \\
\Psi_{2}(\zeta):= & r(\zeta) A\left(z^{\prime}+\zeta\right) A\left(z^{\prime}\right) A\left(z_{1}\right) \ldots A\left(z_{k}\right) \Omega \\
& \left(-\operatorname{Im} \zeta \in \mathscr{V}_{+}\right) .
\end{aligned}
$$

According to the theorem of Section 3 there exists a real neighborhood $\mathscr{V}$ of 0 such that

$$
\left(\Phi_{n-k-1}\left(\bar{z}_{n}, \bar{z}_{n-1}, \ldots, \bar{z}_{k+1}\right), \Psi_{1,2}(\zeta)\right)
$$

can be analytically continued to the points $\zeta \in \mathscr{V}$ if

$$
\begin{aligned}
& \operatorname{Im}\left(z_{j+1}-z_{j}\right) \in \mathscr{V}_{+} \quad(j=k+1, \ldots, n-1) \\
& \operatorname{Im}\left(z^{\prime}-z_{k+1}\right) \in \mathscr{V}_{+} .
\end{aligned}
$$

In Appendix 2 we show that the boundary values

$$
\Psi_{1}(\xi+i 0), \quad \Psi_{2}(\xi-i 0)
$$

exist as distributions over $\mathscr{D}\left(\mathscr{U}_{r}(0)\right)$.

If we now choose a $g \in \mathscr{D}\left(\mathscr{U}_{r}(0) \cap \mathscr{V}\right)$ and define for $\eta \in \mathscr{V}_{+}$, $\operatorname{Im}\left(z_{1}-z^{\prime}\right)-\eta \in \mathscr{V}_{+}$

$$
\begin{aligned}
\Psi_{\eta} & :=\int \Psi_{1}(\xi+i \eta) g(\xi) d \xi \\
\Psi_{-\eta} & :=\int \Psi_{2}(\xi-i \eta) g(\xi) d \xi \\
\Psi_{+} & :=\lim _{\eta \rightarrow 0, \eta \in \mathscr{V}_{+}} \Psi_{\eta} \\
\Psi_{-} & :=\lim _{\eta \rightarrow 0, \eta \in \mathscr{V}_{+}} \Psi_{-\eta}
\end{aligned}
$$

we can show that for each $\Psi \in \mathfrak{H}$

$$
\left(\Psi, \Psi_{+}-\Psi_{-}\right)=0
$$

in the same manner as in the proof of Lemma 1 of Section 3.

From the edge of the wedge theorem we can conclude that there exists a vector-valued holomorphic continuation $\Psi(\zeta)$ of $\Psi_{1}(\zeta)$ and $\Psi_{2}(\zeta)$ to the points $\zeta \in \mathscr{V} \cap \mathscr{U}_{r}(0)$. If we define $\chi:=-\frac{1}{2} \zeta ; z=z^{\prime}-\chi$ we get

$$
\Psi(\zeta)=\hat{\Psi}(\chi)=r(-2 \chi) A(z+\chi) A(z-\chi) A\left(z_{1}\right) \ldots A\left(z_{k}\right) \Omega .
$$

If we divide the Taylor expansion of $\hat{\Psi}(\chi)$ (at $\chi=0)$ by $r(-2 \chi)$ we get the short distance expansion in a similar manner as in the proof of Lemma 2, but now in the sense of operators on $\mathfrak{I}_{y}$.

A remarkable point in the content of lemma is the fact that the short distance expansion is not given for $A(x+\chi) A(x-\chi)$ ( $x$ real) but for $A(z+\chi) A(z-\chi)$, with $z \in \tau_{+}^{(1)}$. Without further assumptions one is not 
allowed to perform the limit $z \rightarrow x$. The resulting operators $B_{k}$ are therefore also not given in the form

but as

$$
B_{k}(f)=\int B_{k}(x) f(x) d x, \quad f \in \mathscr{S}\left(\mathbb{R}^{4}\right)
$$

$$
B_{k}(z)=\int e^{i p z} \tilde{B}_{k}(p) d p \quad(k=1,2,3, \ldots) .
$$

Therefore one is not able to express locality properties of $B_{k}$ by using test functions with compact support in coordinate-space. Especially the relative locality of $B_{k}$ with respect to $A(x)$ appears in the form that the Wightman functions constructed with a $B_{k}(z)$ and other field operators have in their domains of holomorphy the same behaviour for permutation of the arguments as if $B_{k}$ and $A$ would be fields relatively local to each other. The "regularizing function" $r$ is of course not uniquely determined; it can be replaced by $\hat{r}(\zeta)=r(\zeta) h(\zeta)$ where $h$ is holomorphic in a neighborhood of 0 and does not vanish for $\zeta=0$. This arbitrariness leads to a transformation of the composite operators $B_{k}$ and the singular functions $s_{k}$ by a triangular matrix and is therefore very similar to the arbitrariness found by Wilson and Zimmermann [3].

It is clear that our results so far can only be regarded as preliminary if one is interested in more realistic situations. Let us make in the following some remarks to generalisations.

a) A generalisation to cases, where "product-operators" appear at more than one place in the $n$-point function seems to us only to be a slight complication, not a true difficulty.

b) For a generalisation of the results from power 2 to higher powers of the field $A(x)$ some new considerations are necessary; for instance in the case of three operators $A\left(x_{1}\right) A\left(x_{2}\right) A\left(x_{3}\right)$ it would be very helpful for an answer if one could find arguments that the behaviour of such a product for $x_{1} \rightarrow x_{2}$ does not depend "too much" on $x_{2}-x_{3}$.

c) The generalisation which seems to us as that of the greatest importance at the moment refers to the assumed structure of the 4-point function. Besides some plausible technical assumptions the essential condition for the validity of the results of the theorem (Section 3) is d):

There exists a function $r$, such that

is analytic in

$$
W_{4}\left(\zeta_{1}, \zeta_{2}, \zeta_{3}\right) r\left(\zeta_{3}\right)
$$

$$
\left(\zeta_{1}, \zeta_{2}\right) \in \tau_{+}^{(2)} \text { and } \zeta_{3} \in \mathscr{U}_{r}(0) .
$$

Instead of this special assumption one would prefer to assume: There exist functions $u_{k}$, such that

$$
W_{4}\left(\zeta_{1}, \zeta_{2}, \zeta_{3}\right)=\sum_{k=1}^{l} G_{k}^{(4)}\left(\zeta_{1}, \zeta_{2}, \zeta_{3}\right) u_{k}\left(\zeta_{3}\right)
$$


with $G_{k}^{(4)}\left(\zeta_{1}, \zeta_{2}, \zeta_{3}\right)$ holomorphic in the points

$$
\left(\zeta_{1}, \zeta_{2}\right) \in \tau_{+}^{(2)}, \quad \zeta_{3} \in \mathscr{U}_{r}(0) .
$$

It is not difficult to formulate some further plausible assumptions to guarantee in this case representations of $W_{n}, n=5,6,7, \ldots$ in the form

$$
W_{n}\left(\zeta_{1}, \ldots, \zeta_{n-1}\right)=\sum_{k=1}^{l} G_{k}^{(n)}\left(\zeta_{1}, \ldots, \zeta_{n-1}\right) u_{k}\left(\zeta_{n-1}\right)
$$

where $G_{k}^{(n)}\left(\zeta_{1}, \ldots, \zeta_{n-1}\right)$ is analytic in the points $\left(\zeta_{1}, \ldots, \zeta_{n-2}\right) \in \tau_{+}^{(n-2)}$, $\zeta_{n-1} \in \tilde{\mathscr{U}}_{r}(0)\left(\tilde{\mathscr{U}}_{r}(0)\right.$ is also a certain real neighborhood of 0$)$.

The second part of the proof to get the analogue of the theorem in Section 3 must lead from the analyticity of

$$
G_{k}^{(n)}\left(\zeta_{1}, \ldots, \zeta_{n-1}\right), \quad k=1, \ldots, l
$$

with $\left(\zeta_{1}, \ldots, \zeta_{n-2}\right) \in \tau_{+}^{(n-2)}, \zeta_{n-1} \in \tilde{\mathscr{U}}_{r}(0)$ to the analyticity of

with

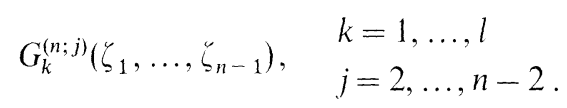

where

$$
\left(\zeta_{1}, \ldots, \zeta_{j-1}, \zeta_{j+1}, \ldots, \zeta_{n-1}\right) \in \tau_{+}^{(n-2)}, \zeta_{j} \in \tilde{\mathscr{U}}_{r}(0) \text {. }
$$

$$
\begin{aligned}
& G_{k}^{(n ; j)}\left(\zeta_{1}, \ldots, \zeta_{n-1}\right) \\
& \quad=G_{k}^{(n)}\left(\zeta_{1}, \ldots, \zeta_{j-2}, \zeta_{j-1}+\zeta_{j}+\zeta_{j+1}, \zeta_{j+2}, \ldots, \zeta_{n-1},-\sum_{l=j}^{n-1} \zeta_{l}, \zeta_{j}\right) .
\end{aligned}
$$

Here lies the true difficulty with respect to the generalisation c). While $W_{n}$ was analytic in all the permuted tubes, it is not clear that the same property holds for $G_{k}^{(n)}, k=1, \ldots, l$, and it is not even known that one can find a neighborhood $\mathscr{W}_{\xi}(0)$ of 0 such that $G_{k}^{(n)}, k=1, \ldots, l$ is analytic for

$$
\left(\zeta_{1}, \ldots, \zeta_{j-1}, \zeta_{j+1}, \ldots, \zeta_{n-1}\right) \in \tau_{+}^{(n-2)}, \zeta_{j} \in \mathscr{W}_{+}(0) \cap\left(\tau_{+}^{(1)} \cup \tau_{-}^{(1)}\right)
$$

( $\mathscr{W}_{-\frac{6}{4}}$ may depend on the other variables).

If one had this last mentioned analyticity property for $G_{k}^{(n)}$ one could get the desired generalisation.

Apart from the further treatment of these generalisations (expressed in the assumption (4.8) - may be also with $l=\infty$ ) a systematic study of the 4-point functions themselves seems to be important in order to see whether an assumption like (4.8) is true in general.

On the other hand the analytic properties expressed in the statement of the theorem of Section 3 can also be used for the light-cone expansion. With this respect we refer to a preprint in preparation. (Authors: J. Kühn and E. Seiler.) 
Acknowledgements. We thank R. Haag, W. Zimmermann, A. S. Wightman, B. Andersson, and M. Karowski (in chronological order) for helpful remarks and discussions, $\mathrm{K}$. Baumann and J. Kühn for correcting some errors in the manuscript.

\section{Appendix 1}

The Separating Character of $\Omega$ for Bilinear Forms

We assume that $B(x)$ is a "bilinear-form-valued tempered distribution" on $\overline{\mathfrak{D}} \times \mathfrak{D}$, that means:

$B$ is a trilinear mapping from $\mathscr{S}\left(\mathbb{R}^{4}\right) \times \overline{\mathfrak{D}} \times \mathfrak{D}$ into $\mathbb{C}$ which is continuous in the first argument (we write $\overline{\mathfrak{D}}$ for the second factor to indicate the antilinearity of $B$ in the second argument). We denote the image of $g \in \mathscr{S}\left(\mathbb{R}^{4}\right) ; \Phi, \Psi \in \mathfrak{D}$ under $B$ by

$$
B(g)(\Phi, \Psi)
$$

and sometimes incorrectly by

$$
(\Phi, B(g) \Psi)
$$

Furthermore we assume that for

$$
\begin{aligned}
\Phi=\Phi_{k}(f)= & \int A\left(x_{1}\right) \ldots A\left(x_{k}\right) f\left(x_{1}, \ldots, x_{k}\right) d x_{1} \ldots d x_{k} \Omega \\
\Psi=\Psi_{l}(h)= & \int A\left(x_{1}\right) \ldots A\left(x_{l}\right) h\left(x_{1}, \ldots, x_{l}\right) d x_{1} \ldots d x_{l} \Omega \\
& \left(f \in \mathscr{S}\left(\mathbb{R}^{4 k}\right), h \in \mathscr{S}\left(\mathbb{R}^{4 l}\right)\right) .
\end{aligned}
$$

$B(g)\left(\Phi_{k}(f), \Phi_{l}(h)\right)$ depends continuously on $f$ and $h$, that means it defines according to the nuclear theorem of Schwartz a tempered distribution $T$ over $\mathscr{S}\left(\mathbb{R}^{4(k+l+1)}\right)$. Finally we assume that $B$ is relatively local to the field $A$ in the weak sense defined in Section 2. Then the following statement holds: If $B().(., \Omega)=0$ then $B=0$.

Proof. The proof is completely analogous to the proof of the separating property of $\Omega$ for local operators (see for instance Ref. [7]). There is only one nontrivial point: One has to show that also in this case the tempered distribution

$$
T\left(f ; g ; y_{1}, \ldots, y_{l}\right):=B(g)\left(\Phi_{k}(f), A\left(y_{1}\right) \ldots A\left(y_{l}\right) \Omega\right)
$$

is the boundary value of a function holomorphic in $\tau_{+}^{(l-1)}$ (we cannot just simply put in the spectral decomposition of $P_{\mu}$ because we could get outside $\mathfrak{D}$, the domain of definition). But we know that $\Phi_{l}(h)=0$ if the support of $h$ lies outside the well-known support of the Fourier-transform of the vector-valued distribution $\Phi_{l}\left(y_{1}, \ldots, y_{l}\right)$; then $B(g)\left(\Phi_{k}(f), \Phi_{l}(h)\right)$ vanishes because of linearity. That means that the Fourier transform of $T\left(f ; g ; y_{1}, \ldots, y_{l}\right)$ with respect to $y_{1}, \ldots, y_{l}$ has the same support properties as $\Phi_{l}\left(p_{1}, \ldots, p_{l}\right)$ and therefore $T$ is boundary value of a function holomorphic in $\tau_{+}^{(l-1)}$. 


\section{Appendix 2}

\section{A Boundary Value Property of Functions Holomorphic in the Forward Tube}

Proposition. Let us assume that $F(\xi+i q)$ is a function holomorphic in $\xi=\xi+i q \in \tau_{+}^{(n)}$ which fulfils for $\xi \in \mathscr{U}$ ( $\mathscr{U}$ is a bounded open set) and each compact subset $\mathscr{K}$ of $\mathscr{V}_{+}$an inequality of the form

$$
|F(\xi+i \eta)|<C_{K}\left(\eta_{1}^{2} \eta_{2}^{2} \ldots \eta_{n}^{2}\right)^{-q}, \quad q>0
$$

for $0<\eta_{j}^{2} \leqq 1 ; \frac{\eta_{j}}{\sqrt{\eta_{j}^{2}}} \in \mathscr{K} \quad(j=1, \ldots, n)^{9}$. Then the boundary value $\lim _{\eta \rightarrow 0 ; \eta_{1} / \sqrt{\eta_{1}^{2}}, \ldots, \eta_{n} / \sqrt{\eta_{n}^{2}} \in \mathscr{K}} F(\xi+i \eta)$ exists in the sense of distributions over $\mathscr{D}(\mathscr{U})$ ( for each $\mathscr{K})$.

Proof. The proof is similar to the proof of Theorem 2.10 in Ref. [7]. Let us define for $g \in \mathscr{D}(\mathscr{U}), 0<\eta_{j}^{2} \leqq 1, \eta_{j} / \sqrt{\eta_{j}^{2}} \in \mathscr{K}(j=1, \ldots, n)$

$$
\begin{gathered}
h(\eta):=\int F(\xi+i \eta) g(\xi) d \xi \\
D^{N} h(\eta)=(-i)^{N} \int F(\xi+i \eta)\left(D^{N} g(\xi)\right) d \xi
\end{gathered}
$$

(we use the Cauchy-Riemann equation for $F$ and partial integration). If we use (A.3) we see that

$$
\left|D_{\eta}^{N} h(\eta)\right| \leqq C_{K}\left(\eta_{1}^{2} \eta_{2}^{2} \ldots \eta_{n}^{2}\right)^{-q} p_{|N|}(g)
$$

where

$$
p_{|N|}(g)=\sup _{\substack{\xi \xi \mathscr{U} \\|j| \leqq|N|}}\left|D_{\xi}^{j} g(\xi)\right| \int_{\mathscr{U}} d \xi\left(N=\left(n_{1}, \ldots, n_{4 n}\right) ;|N|=n_{1}+\cdots+n_{4 n}\right) .
$$

Without loss of generality we can assume that $\hat{\eta}=\left(\hat{\eta}_{1}, \ldots, \hat{\eta}_{n}\right)$ with $\hat{\eta}_{j}=(1,0,0,0)(j=1, \ldots, n)$ is contained in $\mathscr{K}$; we consider the Taylor expansion of $D h(\eta)$ around $\hat{z}$ in the following form due to Cauchy (see Ref. [12], p. 118)

$$
\begin{aligned}
& D h(\eta)=t_{k-1}(\hat{\eta})+\frac{1}{(k-1) !} \int_{0}^{1} d \lambda(1-\lambda)^{k-1} \\
& \cdot\left(\frac{d}{d \lambda}\right)^{k} \operatorname{Dh}(\hat{\eta}+\lambda(\eta-\hat{\eta}))
\end{aligned}
$$

$\overline{{ }^{9}}$ It is reasonable only to consider compact sets $\mathscr{K}$ which contain points $\eta$ with $\eta^{2}=1$. 
$\left(t_{k-1}(\hat{\eta})\right)$ denotes the Taylor expansion of $D h(\eta)$ up to $(k-1)$ th order). If we choose $k>2 n q$ it follows

$$
\begin{aligned}
|D h(\eta)| \leqq & \left|t_{k-1}(\hat{\eta})\right|+\frac{1}{(k-1) !} \int_{0}^{1} d \lambda(1-\lambda)^{k-1} \\
& \cdot\left[\left(\hat{\eta}_{1}+\lambda\left(\eta_{1}-\hat{\eta}_{1}\right)\right)^{2} \ldots\left(\hat{\eta}_{n}+\lambda\left(\eta_{n}-\hat{\eta}_{n}\right)\right)^{2}\right]^{-q} C_{K} p_{k+1}(g) \\
\leqq & \left|t_{k-1}(\hat{\eta})\right|+\frac{1}{(k-1) !} \int_{0}^{1} d \lambda(1-\lambda)^{k-1-2 n q} C_{K} p_{k+1}(g) \\
= & c_{1}(g)+c_{2}(g) C_{K}
\end{aligned}
$$

$\left(c_{1}, c_{2}\right.$ are some constants depending on $\left.g\right)$. Therefore (according to the mean value theorem)

$$
\left|h(\eta)-h\left(\eta^{\prime}\right)\right| \leqq\left|\eta-\eta^{\prime}\right|\left(c_{1}(g)+c_{2}(g) C_{K}\right) \sqrt{2} .
$$

This shows that the limit of $h(\eta)$ for $\eta \rightarrow 0\left(\eta_{j} / \sqrt{\eta_{j}^{2}} \in \mathscr{K} ; 0<\eta_{j}^{2} \leqq 1\right)$ exists for each $g \in \mathscr{D}(\mathscr{U})$ which proves our proposition.

\section{Applications}

1) Multiplication of distributions. Two functions $F_{1}, F_{2}$, fulfilling the assumptions of the proposition can be multiplied; the product fulfils again the assumptions and has therefore a boundary value in the sense of $\mathscr{D}^{\prime}(\mathscr{U})$, which can be interpreted as the product of the boundary values of $F_{1}$ and $F_{2}$. If a function $F(\xi+i \eta)$ is the Laplace transform of a tempered distribution with support in $\overline{\mathscr{V}}_{+}^{n}$, it fulfils the condition (A.3) according to Theorem 2.10 of Ref. [7]. Therefore the product $r\left(\zeta_{j}\right) W_{n}(\xi)$ which occurs in Section 3 has a boundary value in the sense of $\mathscr{D}^{\prime}\left(\mathscr{U}_{r}(0)\right)$. $\left(\mathscr{D}^{\prime}(\mathscr{U})\right.$ denotes the dual space of $\mathscr{D}(\mathscr{U})$.)

2) The vector valued holomorphic function (cf. Section 3 and 4)

$$
\begin{gathered}
\Psi(\zeta):=r(\zeta) A(z) A(z+\zeta) A\left(z_{1}\right) \ldots A\left(z_{k}\right) \Omega \\
\left(\operatorname{Im} z \in \mathscr{V}_{+}, \operatorname{Im} \zeta \in \mathscr{V}_{+}, \operatorname{Im}\left(z_{1}-z-\zeta\right) \in \mathscr{V}_{+},\right. \\
\left.\operatorname{Im}\left(z_{j}-z_{j-1}\right) \in \mathscr{V}_{+} \quad(j=2, \ldots, k)\right)
\end{gathered}
$$

has for $\operatorname{Im} \zeta=\eta \rightarrow 0$ a boundary value in the sense of vector valued distributions over $\mathscr{D}\left(\mathscr{U}_{r}(0)\right)$.

Proof. For $g \in \mathscr{D}\left(\mathscr{U}_{r}(0)\right)$ we define

$$
\Psi_{g}(\eta):=\int \Psi(\xi+i \eta) g(\xi) d \xi \quad\left(\eta \in \mathscr{V}_{+}\right) .
$$

Then

$$
\begin{aligned}
& \left\|\Psi_{g}(\eta)-\Psi_{g}\left(\eta^{\prime}\right)\right\|^{2}=\left(\Psi_{g}(\eta), \Psi_{g}(\eta)\right) \\
& +\left(\Psi_{g}\left(\eta^{\prime}\right), \Psi_{g}\left(\eta^{\prime}\right)\right)-\left(\Psi_{g}(\eta), \Psi_{g}\left(\eta^{\prime}\right)\right) \\
& -\left(\Psi_{g}\left(\eta^{\prime}\right), \Psi_{g}(\eta)\right) .
\end{aligned}
$$


Each term of (A.12) converges for $\eta, \eta^{\prime} \rightarrow 0 ; \frac{\eta}{\sqrt{\eta^{2}}}, \frac{\eta^{\prime}}{\sqrt{\eta^{\prime 2}}} \in \mathscr{K}$ according to our proposition to the same limit, since it is a product of a Wightman function with $r(\zeta)$ and $\overline{r(\zeta)}$ (the latter can be considered as a holomorphic function of $\left.\bar{\zeta} \in \tau_{+}^{(1)}\right)$. Hence $\Psi_{g}(\eta)$ converges strongly for $\eta \rightarrow 0\left(\frac{\eta}{\sqrt{\eta^{2}}} \in \mathscr{K}\right)$.

\section{References}

1. Wilson, K.: On Products of Field Operators at Short Distances. Cornell Report (1964); Phys. Rev. 179, 1499 (1969).

2. Zimmermann, W.: Commun. math. Phys. 6, 161 (1967); 10, 325 (1968). Brandeis lectures Vol. I. Cambridge: MIT Press 1971.

3. Wilson, K., Zimmermann, W.: Commun. math. Phys. 24, 87 (1972).

4. Otterson, P., Zimmermann, W.: Commun. math. Phys. 24, 107 (1972).

5. Brandt, R. A.: Ann. Phys. (N.Y.) 44, 221 (1967); 52, 122 (1969); Fortschr. Phys. 18, 249 (1970).

6. Lowenstein, J. H.: Commun. math. Phys. 16, 265 (1970).

7. Streater,R.F., Wightman, A.S.: PCT, Spin and Statistics and All That. New York: 1964.

8. Jost, R.: The General Theory of Quantized Fields. Providence (AMS), 1965.

9. Vladımirov, V.S.: Methods of the Functions of Several Complex Variables. Cambridge: MIT Press, 1966.

10. Tomozawa. Y.: J. Math. Phys. 4, 1240 (1968) (Theorem 5).

11. De Mottoni, P.. Genz, H.: Nuovo Cim. 67 B, 1 (1970); Nucl. Phys. B 25, 173 (1971).

12. Gel'fand, I.M., Schilow, G.E.: Verallgemeinerte Funktionen (Distributionen). Berlin: 1967.

S. Schlieder, E. Seiler MPI für Physık und Astrophysik

D-8000 München 23

Föhringer Ring 6

Federal Republic of Germany 
